\title{
Domain Decomposition Methods for Space Fractional Partial Differential Equations*
}

\author{
Yingjun Jiang ${ }^{\dagger}$ and Xuejun $\mathrm{Xu}^{\ddagger}$
}

\begin{abstract}
In this paper, a two-level additive Schwarz preconditioner is proposed for solving the algebraic systems resulting from the finite element approximations of space fractional partial differential equations (SFPDEs). It is shown that the condition number of the preconditioned system is bounded by $C(1+H / \delta)$, where $H$ is the maximum diameter of subdomains and $\delta$ is the overlap size among the subdomains. Numerical results are given to support our theoretical findings.
\end{abstract}

Keywords. fractional differential equations, overlapping domain decompositions, preconditioners

\section{Introduction}

Space fractional partial differential equations have been wildly used to describe the supperdiffusion processes in the natural world (see [19]). Let $\Omega$ denote a polyhedral domain in $\mathbb{R}^{d}$ and $\tilde{M}(z)$ denotes a probability density function on $S^{d-1}$, where $S^{d-1}=\left\{z \in \mathbb{R}^{d} ;\|z\|_{2}=1\right\}$ and $\|\cdot\|_{2}$ denotes the standard Euclidean norm. In this paper, we consider the following multi-dimensional $\operatorname{SFPDE}([15])$

$$
-\int_{S^{d-1}} D_{z}^{2 \alpha} u(x) \tilde{M}(z) d z+c u(x)=f(x), \quad x \in \Omega,
$$

where $1 / 2<\alpha<1, c \geq 0$ and $D_{z}^{2 \alpha}$, which will be given later, denotes the directional derivative of order $2 \alpha$ in the direction $z$. We assume $\tilde{M}$ is symmetric about origin, i.e., $\tilde{M}(z)=\tilde{M}\left(z^{\prime}\right)$ if $z, z^{\prime} \in S^{d-1}$ satisfy $z+z^{\prime}=0$, which means that the above SFPDE is symmetric.

Actually, the equation (1.1) is an appropriate extension from one dimensional problem

$$
-\left(p_{-\infty} D_{x}^{2 \alpha}+q_{x} D_{\infty}^{2 \alpha}\right) u+c u=f,
$$

and its corresponding developing equation can be used to describe a general super-diffusion process (see [15] for details), where ${ }_{-\infty} D_{x}^{2 \alpha},{ }_{x} D_{\infty}^{2 \alpha}$ denote Riemann-Liouville fractional derivatives. One

\footnotetext{
${ }^{*}$ The work of the first author is supported by National Natural Science Foundation of China (No. 10901027). The work of second author is supported by the National Basic Research Program under the Grant 2011CB30971 and National Natural Science Foundation of China (No. 11171335, 11225107).

${ }^{\dagger}$ Department of Mathematics and Scientific Computing, Changsha University of Science and Technology, Changsha, 410076, China (jiangyingjun@csust.edu.cn).

${ }^{\ddagger}$ Institute of Computational Mathematics and Scientific/Engineering Computing, Academy of Mathematics and Systems Science, Chinese Academy of Sciences, P.O. Box 2719, Beijing, 100190, P.R. China (xxj@lsec.cc.ac.cn).
} 
special case of (1.1) is

$$
-\sum_{i=1}^{d}\left(p_{i-\infty} D_{x_{i}}^{2 \alpha}+q_{i x_{i}} D_{\infty}^{2 \alpha}\right) u+c u=f
$$

with $p_{i}, q_{i} \in \mathbb{R}$ satisfying $p_{i}=q_{i}$ and $p_{i}+q_{i}=1$. We may find that equation (1.3) can be obtained from (1.1) by taking

$$
\tilde{M}=\sum_{i=1}^{d} p_{i} \delta\left(z-e_{i}\right)+q_{i} \delta\left(z+e_{i}\right),
$$

where $e_{i}$ is the $i$ th column of identity matrix in $\mathbb{R}^{d \times d}$ and $\delta$ denotes the Dirac function on $S^{d-1}$.

Extensive numerical methods have already been developed for SFPDEs, like finite difference methods (see e.g., [2, 17, 18, 25, 26]), finite element methods (see e.g., [6 8, 14, 22]), and spectral methods [11]. Due to the nonlocal properties of fractional differential operators, the most important issue for numerical computation of SFPDEs is how to reduce the computation costs. Some methods for the reduction have already been designed, like alternating-direction implicit methods (ADI) [18,30,31], special iterative methods [12,20,31,34] and multigrid methods [20,36].

The discrete systems $A x=b$ of SFPDEs usually have the following characteristics: 1 ). the condition number of $A$ increases fast, as the mesh becomes fine; 2). the coefficient matrix $A$ is dense. As for iterative methods, two issues need to be concerned for efficiency: one is how to construct good preconditioners for the discrete system $A x=b$, which may help us to save the iterative steps; the other is how to reduce the computation cost of each iterative step, for which some papers employ the multiplication of Toeplitz matrices and vectors with $n \log (n)$ computation complexity (see e.g., [12, 20, 29, 31, 34]). If the iterative step can be carried out in parallel, the efficiency of solving SFPDEs may be significantly improved. The parallelizable algorithms have been wildly used in numerical solutions for PDEs (see e.g. [28). Whereas, to the best of our knowledge, no parallelizable algorithms have been designed for SFPDEs.

In this paper, we shall construct a two-level additive preconditioner for the discrete system $A x=b$ resulting from the finite element approximation of (1.1), and then use the preconditioned conjugate method (PCG) to solve it. The preconditioner we construct is almost optimal, i.e., the condition number of the preconditioned system is bounded by $C(1+H / \delta)$, where $H$ is the maximum diameter of subdomains and $\delta$ is the overlap size among the subdomains. Moreover, the preconditioner may be employed in parallel or each step of the PCG can be carried out in parallel. As a result, the whole numerical solution processes, including the generation of $A, b$ and the multiplication of matrices and vectors, may be conducted in parallel.

Without loss of generality, we focus on the case $d=2$, namely, we consider the problem (1.1) in $\mathbb{R}^{2}$. For $\Lambda \subset \mathbb{R}^{2}$, denote $L^{2}(\Lambda)$ the space of all measurable function $v$ on $\Lambda$ satisfying $\int_{\Lambda}(v(x))^{2} d x<\infty$, and $C_{0}^{\infty}(\Lambda)$ the space of infinitely differentiable functions with compact support in $\Lambda$. Set

$$
(v, w)_{\Lambda}=\iint_{\Lambda} v w d x d y, \quad\|v\|_{\Lambda}=(v, v)_{\Lambda}^{1 / 2}
$$

and they are abbreviated as $(v, w)$ and $\|v\|$ respectively if $\Lambda=\mathbb{R}^{2}$.

To simplify our statement, we make a convention here: function $v$ defined on a domain $\Lambda \subset \mathbb{R}^{2}$ also denotes its extension on $\mathbb{R}^{2}$ which extends $v$ by zero outside $\Lambda$. The constant $C$ with or without subscript shall denote a generic positive constant which may take on different values in different places. These constants shall always be independent of mesh sizes and numbers of subdomains. Following [35], we also use symbols $\lesssim, \gtrsim$ and $\approx$ in this paper. That $a_{1} \lesssim b_{1}, a_{2} \gtrsim b_{2}$ and $a_{3} \approx b_{3}$ 
mean that $a_{1} \leq C_{1} b_{1}, a_{2} \geq C_{2} b_{2}$ and $C_{3} b_{3} \leq a_{3} \leq C_{3}^{\prime} b_{3}$ for some positive constants $C_{1}, C_{2}, C_{3}$ and $C_{3}^{\prime}$.

The rest of the paper is organized as follows: in section 2, the variational problem of (1.1) and its finite element discretization are described; in section 3, the two-level additive preconditioner for the SFPDEs is presented; in section 4, it is proved that the preconditioner is almost optimal; finally, in section 5 , the numerical results shall be given to support our theoretical findings.

\section{The model problem and its discretization}

In this section, we shall describe the SFPDEs in details, and then introduce its variational formulation and finite element discretization.

\subsection{The model problem}

Definition 2.1. 8 ] Let $\mu>0, \theta \in \mathbb{R}$. The $\mu$ th order fractional integral in the direction $z=$ $(\cos \theta, \sin \theta)$ is defined by

$$
D_{z}^{-\mu} v(x, y):=D_{\theta}^{-\mu} v(x, y)=\int_{0}^{\infty} \frac{\tau^{\mu-1}}{\Gamma(\mu)} v(x-\tau \cos \theta, y-\tau \sin \theta) d \tau,
$$

where $\Gamma$ is the Gamma function.

Definition 2.2. [8] Let $n$ be a positive integer, and $\theta \in \mathbb{R}$. The $n$th order derivative in the direction of $z=(\cos \theta, \sin \theta)$ is given by

$$
D_{\theta}^{n} v(x, y):=\left(\cos \theta \frac{\partial}{\partial x}+\sin \theta \frac{\partial}{\partial y}\right)^{n} v(x, y) .
$$

Definition 2.3. 8] Let $\mu>0, \theta \in \mathbb{R}$. Let $n$ be the integer such that $n-1 \leq \mu<n$, and define $\sigma=n-\mu$. Then the $\mu$ th order directional derivative in the direction of $z=(\cos \theta, \sin \theta)$ is defined by

$$
D_{z}^{\mu} v(x, y):=D_{\theta}^{\mu} v(x, y)=D_{\theta}^{n} D_{\theta}^{-\sigma} v(x, y) .
$$

If $v$ is viewed as a function in $x, D_{0}^{\mu}, D_{\pi}^{\mu}$ are just the left and the right Riemamm-Liouville derivatives (see Appendix).

Definition 2.4. 8] Assume that $v: \mathbb{R}^{2} \rightarrow \mathbb{R}, \mu>0$. The $\mu$ th order fractional derivative with respect to the measure $\tilde{M}$ is defined as

$$
D_{\tilde{M}}^{\mu} v(x, y):=\int_{S^{1}} D_{\theta}^{\mu} v(x, y) \tilde{M}(\theta) d \theta
$$

where $S^{1}=[0+\nu, 2 \pi+\nu)$ with a suitable scalar $\nu$, and $\tilde{M}(\theta)$, which satisfies $\int_{\nu}^{2 \pi+\nu} \tilde{M}(\theta) d \theta=1$, is a periodic function with period $2 \pi$. Usually we take $\nu=0$, if it causes no unreasonable expression (see (1.4) ).

For $u: \mathbb{R}^{2} \rightarrow \mathbb{R}$, define differential operator $L_{\alpha}$ in $\mathbb{R}^{2}$ as

$$
L_{\alpha} u=-D_{\tilde{M}}^{2 \alpha} u+c u \text {. }
$$


Denote $\Omega$ a polygonal domain in $\mathbb{R}^{2}$, set $1 / 2<\alpha<1$. The model problem of this paper is to find $u: \bar{\Omega} \rightarrow \mathbb{R}$ such that

$$
\left\{\begin{array}{cc}
L_{\alpha} u=f, & \text { in } \Omega, \\
u=0, & \text { on } \partial \Omega,
\end{array}\right.
$$

where $f$ is a source term and we assume that $\tilde{M}(\theta)$ satisfies $\tilde{M}(\theta)=\tilde{M}(\theta+\pi)$ for $\theta \in \mathbb{R}$, i.e., (2.1) is a symmetric problem. Here, we recall the convection made in Section 1; $u$ also denotes its extension by zero outside $\Omega$.

\subsection{The variational formulation and finite element discretization}

Definition 2.5. 27] Let $\mu \geq 0, \mathcal{F} v\left(\xi_{1}, \xi_{2}\right)$ be the Fourier transform of $v(x, y),|\xi|=\sqrt{\xi_{1}^{2}+\xi_{2}^{2}}$. Define norm

$$
\|v\|_{H^{\mu}\left(\mathbb{R}^{2}\right)}:=\left\|\left(1+|\xi|^{2}\right)^{\mu / 2}|\mathcal{F} v|\right\| .
$$

Let $H^{\mu}\left(\mathbb{R}^{2}\right):=\left\{v \in L^{2}\left(\mathbb{R}^{2}\right) ;\|v\|_{H^{\mu}\left(\mathbb{R}^{2}\right)}<\infty\right\}$.

For $v \in H_{0}^{\mu}(\Omega)$, we also denote $\|v\|_{H^{\mu}\left(\mathbb{R}^{2}\right)}$ by $\|v\|_{H^{\mu}(\Omega)}$. It is known that $H^{\mu}\left(\mathbb{R}^{2}\right)$ is a Hilbert space equipped with the inner product $(v, w)_{H^{\mu}\left(\mathbb{R}^{2}\right)}=\left(\left(1+|\xi|^{2}\right)^{\mu} \mathcal{F} v, \overline{\mathcal{F} w}\right)$ and $C_{0}^{\infty}\left(\mathbb{R}^{2}\right)$ is dense in $H^{\mu}\left(\mathbb{R}^{2}\right)$ (see [27]). Since we employ the finite element discretization, the weak fractional directional derivative need to be introduced. Let $L_{l o c}^{1}\left(\mathbb{R}^{2}\right)$ denote the set of locally integrable functions on $\mathbb{R}^{2}$.

Definition 2.6. 9] Given $\mu>0, \theta \in \mathbb{R}$, let $v \in L^{2}\left(\mathbb{R}^{2}\right)$. If there is a function $v_{\mu} \in L_{l o c}^{1}\left(\mathbb{R}^{2}\right)$ such that

$$
\left(v, D_{\theta+\pi}^{\mu} w\right)=\left(v_{\mu}, w\right), \quad \forall w \in C_{0}^{\infty}\left(\mathbb{R}^{2}\right),
$$

then $v_{\mu}$ is called the weak $\mu$ th order derivative in the direction of $\theta$ for $v$, denoted by $D_{\theta}^{\mu} v$, i.e., $v_{\mu}=D_{\theta}^{\mu} v$.

The weak derivative $D_{\theta}^{\mu} v$ is unique if it exists and the weak derivative coincides with the correspondent derivative defined in Definition 2.3 if $v \in C_{0}^{\infty}\left(\mathbb{R}^{2}\right)$. In the following, we use $D_{\theta}^{\mu} v$ to denote the weak derivative.

Lemma 2.7. [8, 9] Let $\mu>0$. For any $v \in H^{\mu}\left(\mathbb{R}^{2}\right), 0<s \leq \mu$ and $\theta \in \mathbb{R}$, the weak derivative $D_{\theta}^{s} v$ exists and satisfies

$$
\begin{gathered}
\mathcal{F} D_{\theta}^{s} v\left(\xi_{1}, \xi_{2}\right)=\left(2 \pi i \xi_{1} \cos \theta+2 \pi i \xi_{2} \sin \theta\right)^{s} \mathcal{F} v\left(\xi_{1}, \xi_{2}\right), \\
\left\|D_{\theta}^{s} v\right\| \leq C\|v\|_{H^{\mu}\left(\mathbb{R}^{2}\right)} .
\end{gathered}
$$

Define the bilinear form $\tilde{B}: H_{0}^{\alpha}(\Omega) \times H_{0}^{\alpha}(\Omega) \rightarrow \mathbb{R}$ as

$$
\tilde{B}(u, v):=-\int_{0}^{2 \pi}\left(D_{\theta}^{\alpha} u, D_{\theta+\pi}^{\alpha} v\right) \tilde{M}(\theta) d \theta+c(u, v) .
$$

Because $\tilde{M}(\theta)=\tilde{M}(\theta+\pi)$ for $\theta \in \mathbb{R}$, it is easy to check that $\tilde{B}(v, w)$ is a symmetric bilinear form, i.e., $\tilde{B}(v, w)=\tilde{B}(w, v)$ for $v, w \in H_{0}^{\alpha}(\Omega)$. The variational formulation of (2.1) (see [8,9]) is to find $u \in H_{0}^{\alpha}(\Omega)$ such that

$$
\tilde{B}(u, v)=(f, v), \quad \forall v \in H_{0}^{\alpha}(\Omega)
$$


Here $\tilde{M}$ is taken such that (2.4) admits a unique solution in $H_{0}^{\alpha}(\Omega)$ (for the details, we refer to $[\underline{8}, 9]$ ).

We construct a quasi-uniform triangulation $\Gamma_{H}=\left\{\Omega_{i}\right\}_{i=1}^{J}$ of $\Omega$ with

the diameter of $\Omega_{i} \approx O(H)$.

Divide each $\Omega_{i}$ into smaller simplices $\tau_{j}$ of diameter $O(h)$, such that $\Gamma_{h}=\left\{\tau_{j}\right\}$ form a finer triangulation of $\Omega$. Denote $V_{H}$ and $V_{h}$ piecewise linear finite element function spaces defined on the triangulations $\Gamma_{H}$ and $\Gamma_{h}$ respectively. It is known that $V_{h} \subset H_{0}^{1}(\Omega) \subset H_{0}^{\alpha}(\Omega)$.

The finite element approximation for (2.4) (the details please see [9]) is to find $u_{h} \in V_{h}$ such that

$$
B\left(u_{h}, v\right)=(f, v), \quad \forall v \in V_{h},
$$

where $B(v, w)=-\int_{0}^{2 \pi}\left(D_{\theta}^{\alpha} v, D_{\theta+\pi}^{\alpha} w\right) M(\theta) d \theta+c(v, w), M(\theta)$ is equal to a discrete form $\sum_{k=1}^{L} p_{k} \delta(\theta-$ $\theta_{k}$ ) such that $B(\cdot, \cdot)$ is a symmetric bilinear form, and

$$
B(v, v) \gtrsim\|v\|_{H^{\alpha}(\Omega)}^{2}, \quad B(v, w) \lesssim\|v\|_{H^{\alpha}(\Omega)}\|w\|_{H^{\alpha}(\Omega)}, \quad v, w \in H_{0}^{\alpha}(\Omega) .
$$

Meanwhile

$$
\int_{0}^{2 \pi} M(\theta) d \theta \lesssim 1
$$

Remark 2.8. The direct finite element discretization of (2.4) is

$$
\tilde{B}\left(u_{h}, v\right)=(f, v), \quad v \in V_{h} .
$$

But the discretization is hardly computed. So we use (2.5) instead of (2.7), where $B(\cdot, \cdot)$ is understood as the approximation to $\tilde{B}(\cdot, \cdot)$. For the details, please refer to $[9]$.

\section{A two-level additive Schwarz preconditioner}

Take $f_{h} \in V_{h}$ such that $\left(f_{h}, v\right)=(f, v), \forall v \in V_{h}$ and define a linear operator $A: V_{h} \rightarrow V_{h}$ satisfying

$$
(A v, w)=B(v, w), \quad \forall v, w \in V_{h} .
$$

Since $B(v, w)$ is a symmetric bilinear form, by (2.6), we know that $A: V_{h} \rightarrow V_{h}$ is symmetric positive definite with respect to $(\cdot, \cdot)$, i.e.,

$$
(A v, w)=(v, A w), \quad v, w \in V_{h} ; \quad(A v, v)>0, \quad 0 \neq v \in V_{h} .
$$

Then bilinear form

$$
(v, w)_{A}:=(A v, w)
$$

also induces an inner product on $V_{h}$. Set norm

$$
\|v\|_{A}=(A v, v)^{1 / 2}, \quad v \in V_{h} .
$$

By (2.6), we have

$$
\|v\|_{A} \approx\|v\|_{H^{\alpha}(\Omega)}, \quad \forall v \in V_{h}
$$


The problem (2.5) can be restated as to find $u_{h} \in V_{h}$ such that

$$
A u_{h}=f_{h} .
$$

For the above equation, we shall construct our two-level Schwarz preconditioner and then use PCG method to solve it.

Our preconditioner is designed by making use of the following overlapping domain decomposition $\Omega=\cup_{i=1}^{J}\left\{\Omega_{i}^{\prime}\right\}$, where the subdomain $\Omega_{i}^{\prime}$ contains coarse subdomain $\Omega_{i}$, and satisfies

$$
\text { the diameter of } \Omega_{i}^{\prime} \approx O(H) \text {. }
$$

Meanwhile the boundary of $\Omega_{i}^{\prime}$ align with the mesh of triangulation $\Gamma_{h}$, and the distance from $\partial \Omega_{i}^{\prime} \cap \Omega$ to $\Omega_{i}$ is greater than $\delta$, which is a positive constant measuring the overlapping size among the subdomians. Define subspaces $V_{i}$ of $V_{h}$ as

$$
V_{i}=\left\{v \in V_{h} \mid v(x)=0, x \in \Omega \backslash \Omega_{i}^{\prime}\right\}, \quad i=1,2, \ldots, J,
$$

and let $V_{0}=V_{H}$.

For our analysis, we regroup the subregions in terms of the coloring strategy (see e.g. [28]). By a minimal or good coloring, we group the subregions $\left\{\Omega_{i}^{\prime}\right\}$ into $J^{C}$ classes, each of which has some disjoint subregions and can be regarded as one subregion. Exactly, decompose the index set $\{1,2, \ldots, J\}=\cup_{i=1}^{J^{C}} I_{i}$ with $I_{i}$ satisfying that $\Omega_{l}^{\prime} \cap \Omega_{k}^{\prime}=\emptyset$ for any $l, k \in I_{i}(l \neq k)$; for $i=1, \ldots, J^{C}$, define new subregions $\tilde{\Omega}_{i}=\cup_{j \in I_{i}} \Omega_{j}^{\prime}$ and new subspaces $\tilde{V}_{i}=\bigoplus_{j \in I_{i}} V_{j}$.

For each $k \in\{0,1,2, \ldots, J\}$, we define some projectors $Q_{k}, P_{k}: V_{h} \rightarrow V_{k}$ by

$$
\left(Q_{k} v, w\right)=(v, w), \quad\left(P_{k} v, w\right)_{A}=(v, w)_{A}, \quad \forall w \in V_{k}, v \in V_{h},
$$

and define the linear operator $A_{k}: V_{k} \rightarrow V_{k}$ by

$$
\left(A_{k} v, w\right)=(A v, w), \quad v, w \in V_{k} .
$$

It is not hard to verify that

$$
A_{k} P_{k}=Q_{k} A, \quad k=0,1, \ldots, J .
$$

To help our analysis, we define some projectors $\tilde{P}_{i}: V_{h} \rightarrow \tilde{V}_{i}, i=1, \ldots, J^{C}$, by

$$
\left(\tilde{P}_{i} v, w\right)_{A}=(v, w)_{A}, \quad \forall w \in \tilde{V}_{i}, v \in V_{h} .
$$

Remark 3.1. Different from the integer order PDEs, it is interesting to see that $\tilde{P}_{i} \neq \sum_{j \in I_{i}} P_{j}$.

Now, we are ready to present our two-level additive Schwarz pre-conditioner, i.e.,

$$
B_{h}=\sum_{i=0}^{J} A_{i}^{-1} Q_{i}
$$

By (3.4), we have

$$
B_{h} A=\sum_{i=0}^{J} A_{i}^{-1} Q_{i} A=\sum_{i=0}^{J} A_{i}^{-1} A_{i} P_{i}=\sum_{i=0}^{J} P_{i} .
$$

Define $P_{h}:=\sum_{i=0}^{J} P_{i}$, and then the preconditioned system is

$$
P_{h} u_{h}=B_{h} f_{h} .
$$

In the next section, we shall prove the condition number of $P_{h}$ is bounded by $C(1+H / \delta)$, where the constant $C$ is independent of mesh size and the numbers of subdomains, but dependent of $J^{C}$. 


\section{Condition number estimate}

We first introduce two interpolation norms and relevant Sobolev spaces (see e.g., [27]). Let $\Lambda$ be a domain in $\mathbb{R}^{2}$. For integer $m$, denote by $\|\cdot\|_{\tilde{H}^{m}(\Lambda)}$ the Sobolev norm of integer order $m$, i.e.,

$$
\|v\|_{\tilde{H}^{m}(\Lambda)}:=\left(\sum_{|l| \leq m}\left\|D^{l} v\right\|_{L^{2}(\Lambda)}^{2}\right)^{1 / 2}
$$

with $l=\left(l_{1}, l_{2}\right),|l|=l_{1}+l_{2}$ and $D^{l}=\left(\frac{d}{d x}\right)^{l_{1}}\left(\frac{d}{d y}\right)^{l_{2}}$. Let $\mu>0$ be a non-integer and $0<s<1, n$ is a non-negative integer such that $n<\mu<n+1$. We introduce the interpolation norms

$$
\|v\|_{\tilde{H}^{\mu}(\Lambda)}:=\left(\int_{0}^{\infty} \tilde{K}(v, t) t^{-2 \mu-1} d t\right)^{1 / 2},\|v\|_{\hat{H}^{s}(\Lambda)}:=\left(\int_{0}^{\infty} \hat{K}(v, t) t^{-2 s-1} d t\right)^{1 / 2},
$$

where

$$
\begin{gathered}
\tilde{K}(v, t):=\inf _{w \in \tilde{H}^{n+1}(\Lambda)}\left(\|v-w\|_{\tilde{H}^{n}(\Lambda)}^{2}+t^{2}\|w\|_{\tilde{H}^{n+1}(\Lambda)}^{2}\right), \\
\hat{K}(v, t):=\inf _{w \in \tilde{H}_{0}^{1}(\Lambda)}\left(\|v-w\|_{L^{2}(\Lambda)}^{2}+t^{2}\|w\|_{\tilde{H}^{1}(\Lambda)}^{2}\right) .
\end{gathered}
$$

Relevant Sobolev spaces are

$$
\tilde{H}^{\mu}(\Lambda):=\left\{v \in L^{2}(\Lambda) ;\|v\|_{\tilde{H}^{\mu}(\Lambda)}<\infty\right\}, \quad \hat{H}^{s}(\Lambda):=\left\{v \in L^{2}(\Lambda) ;\|v\|_{\hat{H}^{s}(\Lambda)}<\infty\right\} .
$$

For $\mu>0$, it is known that $\tilde{H}^{\mu}\left(\mathbb{R}^{2}\right)$ coincides with $H^{\mu}\left(\mathbb{R}^{2}\right)$. The following norms relation is useful in our analysis.

Lemma 4.1. [13, 27] Let $0<\mu<1$ with $\mu \neq 1 / 2$ and $\Lambda$ be a domain in $\mathbb{R}^{2}$ with Lipschitz boundary. Then $\hat{H}^{\mu}(\Lambda)$ coincides with $\tilde{H}_{0}^{\mu}(\Lambda)$ with equivalent norms.

In the following, we shall give some useful results.

Lemma 4.2. If $\operatorname{dist}\left(\Omega_{i}^{\prime}, \Omega_{j}^{\prime}\right) \geq l H$ for integer $l \geq 1$, we have

$$
\left(D_{\theta}^{\alpha} v, D_{\theta+\pi}^{\alpha} w\right) \lesssim \frac{1}{l^{0.5+\alpha}}\|v\|_{A}\|w\|_{A}, \quad v \in V_{i}, w \in V_{j} .
$$

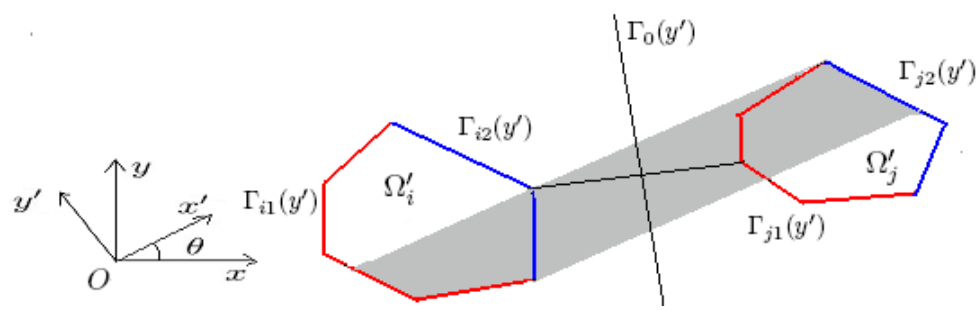

Figure 1: 
Proof. Without loss of generality, we prove that (4.3) holds under the situation as the figure 1 shows. Cartesian coordinate $x^{\prime} O y^{\prime}$ is obtained by rotating $x O y \theta$ angle counterclockwise. We set that $p_{i} \in \bar{\Omega}_{i}$ and $p_{j} \in \bar{\Omega}_{j}$ such that the length of line segment $p_{i} p_{j}$ is equal to $\operatorname{dist}\left(\Omega_{i}^{\prime}, \Omega_{j}^{\prime}\right)$, the graphs of functions $x^{\prime}=\Gamma_{i 1}\left(y^{\prime}\right)$ and $x^{\prime}=\Gamma_{i 2}\left(y^{\prime}\right)$ are parts of boundary $\partial \Omega_{i}^{\prime}$ such that $\Omega_{i}^{\prime}=$ $\left\{\left(x^{\prime}, y^{\prime}\right): \Gamma_{i 1}\left(y^{\prime}\right)<x^{\prime}<\Gamma_{i 2}\left(y^{\prime}\right)\right\}$, the graphs of functions $\Gamma_{j 1}\left(y^{\prime}\right)$ and $\Gamma_{j 2}\left(y^{\prime}\right)$ are parts of boundary $\partial \Omega_{j}^{\prime}$ such that $\Omega_{j}^{\prime}=\left\{\left(x^{\prime}, y^{\prime}\right): \Gamma_{j 1}\left(y^{\prime}\right)<x^{\prime}<\Gamma_{j 2}\left(y^{\prime}\right)\right\}$, and the graph of $x^{\prime}=\Gamma_{0}\left(y^{\prime}\right)$ is the perpendicular bisector of $p_{i} p_{j}$. Then we have $D_{\theta}^{\alpha}={ }_{-\infty} D_{x^{\prime}}^{\alpha}, D_{\theta+\pi}^{\alpha}={ }_{x^{\prime}} D_{\infty}^{\alpha}$, where ${ }_{-\infty} D_{x^{\prime}}^{\alpha}$ and ${ }_{x^{\prime}} D_{\infty}^{\alpha}$ are the left and right Riemann-Liouville fractional derivative operator (see Appendix). We in the following only prove (4.3) for the case that there exists a ray in $(\cos \theta, \sin \theta)$ direction going through both $\Omega_{i}^{\prime}$ and $\Omega_{j}^{\prime}$ from $\Omega_{i}^{\prime}$ to $\Omega_{j}^{\prime}$. Indeed, when the other case is true, $\left(D_{\theta}^{\alpha} v, D_{\theta+\pi}^{\alpha} w\right)=0$ and (4.3) naturally holds.

Denote

$$
\begin{aligned}
& M_{y^{\prime}}=\max _{\substack{\left(x^{\prime}, y^{\prime}\right) \in \Omega_{i}^{\prime} \\
\left(x^{\prime \prime}, y^{\prime}\right) \in \Omega_{j}^{\prime}}} y^{\prime}, \quad m_{y^{\prime}}=\min _{\substack{\left(x^{\prime}, y^{\prime}\right) \in \Omega_{i}^{\prime} \\
\left(x^{\prime \prime}, y^{\prime}\right) \in \Omega_{j}^{\prime}}} y^{\prime}, \\
& M_{x^{\prime}}=\max _{\left(x^{\prime}, y^{\prime}\right) \in \Omega_{j}^{\prime}} x^{\prime}, \quad m_{x^{\prime}}=\min _{\left(x^{\prime}, y^{\prime}\right) \in \Omega_{i}^{\prime}} x^{\prime} .
\end{aligned}
$$

The shaded area is

$$
\Lambda=\left\{\left(x^{\prime}, y^{\prime}\right) \mid \Gamma_{i, 1}\left(y^{\prime}\right) \leq x^{\prime} \leq \Gamma_{j, 2}\left(y^{\prime}\right), m_{y^{\prime}} \leq y^{\prime} \leq M_{y^{\prime}}\right\} .
$$

Let $\Lambda_{i}=\left\{\left(x^{\prime}, y^{\prime}\right) \mid x^{\prime} \leq \Gamma_{0}\left(y^{\prime}\right)\right\} \cap \Lambda$ and $\Lambda_{j}=\left\{\left(x^{\prime}, y^{\prime}\right) \mid x^{\prime} \geq \Gamma_{0}\left(y^{\prime}\right)\right\} \cap \Lambda$. Then we have

$$
\begin{aligned}
\left(D_{\theta}^{\alpha} v, D_{\theta+\pi}^{\alpha} w\right) & =\left(D_{\theta}^{\alpha} v, D_{\theta+\pi}^{\alpha} w\right)_{\Lambda}=\left(D_{\theta}^{\alpha} v, D_{\theta+\pi}^{\alpha} w\right)_{\Lambda_{i}}+\left(D_{\theta}^{\alpha} v, D_{\theta+\pi}^{\alpha} w\right)_{\Lambda_{j}} \\
& \leq\left\|D_{\theta}^{\alpha} v\right\|_{\Lambda_{i}}\left\|D_{\theta+\pi}^{\alpha} w\right\|_{\Lambda_{i}}+\left\|D_{\theta}^{\alpha} v\right\|_{\Lambda_{j}}\left\|D_{\theta+\pi}^{\alpha} w\right\|_{\Lambda_{j}} .
\end{aligned}
$$

For $(x, y) \in \Lambda_{j}$ whose coordinate under $x^{\prime} O y^{\prime}$ is $\left(x^{\prime}, y^{\prime}\right)$, noting that $\operatorname{supp}(v) \subset \Omega_{i}^{\prime}$, we have

$$
\begin{aligned}
D_{\theta}^{\alpha} v(x, y) & =-\infty D_{x^{\prime}}^{\alpha} v\left(x^{\prime}, y^{\prime}\right)=\frac{d}{d x^{\prime}}-\infty D_{x^{\prime}}^{-(1-\alpha)} v\left(x^{\prime}, y^{\prime}\right) \\
& =\frac{1}{\Gamma(1-\alpha)} \frac{d}{d x^{\prime}} \int_{-\infty}^{x^{\prime}}\left(x^{\prime}-s\right)^{-\alpha} v\left(s, y^{\prime}\right) d s \\
& =\frac{1}{\Gamma(1-\alpha)} \frac{d}{d x^{\prime}} \int_{\Gamma_{i 1}\left(y^{\prime}\right)}^{\Gamma_{i 2}\left(y^{\prime}\right)}\left(x^{\prime}-s\right)^{-\alpha} v\left(s, y^{\prime}\right) d s \\
& =\frac{1}{\Gamma(-\alpha)} \int_{\Gamma_{i 1}\left(y^{\prime}\right)}^{\Gamma_{i 2}\left(y^{\prime}\right)}\left(x^{\prime}-s\right)^{-(1+\alpha)} v\left(s, y^{\prime}\right) d s \\
& \lesssim(l H)^{-(1+\alpha)} \int_{\Gamma_{i 1}\left(y^{\prime}\right)}^{\Gamma_{i 2}\left(y^{\prime}\right)}\left|v\left(s, y^{\prime}\right)\right| d s \\
& \lesssim(l H)^{-(1+\alpha)} H^{1 / 2}\left(\int_{\Gamma_{i 1}\left(y^{\prime}\right)}^{\Gamma_{i 2}\left(y^{\prime}\right)} v^{2}\left(s, y^{\prime}\right) d s\right)^{1 / 2}
\end{aligned}
$$

where in the fifth equality we have used the relation $\Gamma(1-\alpha)=-\alpha \Gamma(-\alpha)$, the first inequality is 
by $\left(x^{\prime}-s\right) \geq l H / 2$ when $s \leq \Gamma_{i 2}\left(y^{\prime}\right)$ and the last by the Cauchy-Schwarz inequality. Then

$$
\begin{aligned}
\left\|D_{\theta}^{\alpha} v\right\|_{\Lambda_{j}}^{2} & \lesssim(l H)^{-2(1+\alpha)} H \int_{m_{x^{\prime}}}^{M_{x^{\prime}}} d x^{\prime} \int_{m_{y^{\prime}}}^{M_{y^{\prime}}} d y^{\prime} \int_{\Gamma_{i 1}\left(y^{\prime}\right)}^{\Gamma_{i 2}\left(y^{\prime}\right)} v^{2}\left(s, y^{\prime}\right) d s \\
& \lesssim(l H)^{-(1+2 \alpha)} H \int_{m_{y^{\prime}}}^{M_{y^{\prime}}} d y^{\prime} \int_{\Gamma_{i 1}\left(y^{\prime}\right)}^{\Gamma_{i 2}\left(y^{\prime}\right)} v^{2}\left(s, y^{\prime}\right) d s \\
& \leq(l H)^{-(1+2 \alpha)} H \iint_{\Omega_{i}^{\prime}} v^{2} d x^{\prime} d y^{\prime}=l^{-(1+2 \alpha)} H^{-2 \alpha}\|v\|_{\Omega_{i}^{\prime}}^{2},
\end{aligned}
$$

where the second inequality is by $\int_{m_{x^{\prime}}}^{M_{x^{\prime}}} d x^{\prime} \lesssim l H$. Denote $d_{i}$ as the diameter of $\Omega_{i}^{\prime}$, and define a function in $x^{\prime}$ as

$$
H_{i}\left(x^{\prime}\right)= \begin{cases}0, & \text { if } x^{\prime}>d_{i} ; \\ \frac{x^{\prime(\alpha-1)}}{\Gamma(\alpha)} & \text { if } 0 \leq x^{\prime} \leq d_{i} .\end{cases}
$$

For $(x, y) \in \Omega_{i}^{\prime}$ (whose coordinate is $\left(x^{\prime}, y^{\prime}\right)$ under $\left.x^{\prime} O y^{\prime}\right)$, by (‥4), we have

$$
v(x, y)=D_{\theta}^{-\alpha} D_{\theta}^{\alpha} v(x, y)={ }_{-\infty} D_{x^{\prime}}^{-\alpha}{ }_{-\infty} D_{x^{\prime}}^{\alpha} v\left(x^{\prime}, y^{\prime}\right)=H_{i} *{ }_{-\infty} D_{x^{\prime}}^{\alpha} v\left(\cdot, y^{\prime}\right),
$$

where $v * w$ denote the convolution product (see e.g., [1]). Then by the Young Theorem (Theorem 4.30 in [1]),

$$
\left\|v\left(\cdot, y^{\prime}\right)\right\|_{\left[\Gamma_{i 1}, \Gamma_{i 2}\right]} \leq\left\|v\left(\cdot, y^{\prime}\right)\right\|_{\mathbb{R}} \leq\left\|H_{i}\right\|_{L^{1}(\mathbb{R})}\left\|_{-\infty} D_{x^{\prime}}^{\alpha} v\left(\cdot, y^{\prime}\right)\right\|_{\mathbb{R}} \lesssim H^{\alpha}\left\|_{-\infty} D_{x^{\prime}}^{\alpha} v\left(\cdot, y^{\prime}\right)\right\|_{\mathbb{R}} .
$$

Furthermore we have

$$
\begin{aligned}
\|v\|_{\Omega_{i}^{\prime}}^{2} & =\iint_{\Omega_{i}^{\prime}} v^{2} d x^{\prime} d y^{\prime}=\int_{m_{y^{\prime}}}^{M_{y^{\prime}}}\left\|v\left(\cdot, y^{\prime}\right)\right\|_{\left[\Gamma_{i 1}\left(y^{\prime}\right), \Gamma_{i 2}\left(y^{\prime}\right)\right]}^{2} d y^{\prime} \\
& \lesssim H^{2 \alpha}\left\|_{-\infty} D_{x^{\prime}}^{\alpha} v\right\|_{\mathbb{R}^{2}}^{2}=H^{2 \alpha}\left\|D_{\theta}^{\alpha} v\right\|_{\mathbb{R}^{2}}^{2} \\
& \lesssim H^{2 \alpha}\|v\|_{H^{\alpha}(\Omega)}^{2} \approx H^{2 \alpha}\|v\|_{A}^{2},
\end{aligned}
$$

where the second inequality is by Lemma 2.7, the last equality is by (3.2). Combining (4.9) with (4.6), we obtain

$$
\left\|D_{\theta}^{\alpha} v\right\|_{\Lambda_{j}} \lesssim \frac{1}{l^{1 / 2+\alpha}}\|v\|_{A}
$$

Similarly we may also obtain

$$
\left\|D_{\theta+\pi}^{\alpha} w\right\|_{\Lambda_{i}} \lesssim \frac{1}{l^{1 / 2+\alpha}}\|w\|_{A}
$$

By Lemma 2.7,

$$
\left\|D_{\theta+\pi}^{\alpha} w\right\|_{\Lambda_{j}} \lesssim\|w\|_{H^{\alpha}(\Omega)} \approx\|w\|_{A}, \quad\left\|D_{\theta}^{\alpha} v\right\|_{\Lambda_{i}} \lesssim\|v\|_{H^{\alpha}(\Omega)} \approx\|v\|_{A} .
$$

Combining with (4.4), (4.10) and (4.11), we may obtain (4.3).

Lemma 4.3. Let $S \subset\{1,2, \ldots, J\}$ denote an index set such that $\Omega_{i}^{\prime} \cap \Omega_{j}^{\prime}=\emptyset$ for any $i, j \in S$, $i \neq j$. Then for $v=\sum_{i \in S} v_{i}$ with $v_{i} \in V_{i}$, we have

$$
(v, v)_{A} \lesssim \sum_{i \in S}\left(v_{i}, v_{i}\right)_{A} .
$$


Proof. Note that

$$
(v, v)_{A}=B(v, v)=-\int_{0}^{2 \pi}\left(D_{\theta}^{\alpha} v, D_{\theta+\pi}^{\alpha} v\right) M(\theta) d \theta+c(u, v),
$$

it is easy to see that the lemma follows after we prove that

$$
\left|\left(D_{\theta}^{\alpha} v, D_{\theta+\pi}^{\alpha} v\right)\right| \leq C \sum_{i \in S}\left(v_{i}, v_{i}\right)_{A}
$$

holds for any $\theta$. So next, we give a proof of (4.14). It is easy to see that

$$
\left|\left(D_{\theta}^{\alpha} v, D_{\theta+\pi}^{\alpha} v\right)\right| \leq \sum_{k, j \in S}\left|\left(D_{\theta}^{\alpha} v_{k}, D_{\theta+\pi}^{\alpha} v_{j}\right)\right| .
$$

In fact, we may prove

$$
\sum_{k, j \in S}\left|\left(D_{\theta}^{\alpha} v_{k}, D_{\theta+\pi}^{\alpha} v_{j}\right)\right| \leq C \sum_{i \in S}\left(v_{i}, v_{i}\right)_{A}
$$

through using the following two kind of inequalities:

$$
\begin{aligned}
\left(D_{\theta}^{\alpha} v_{k}, D_{\theta+\pi}^{\alpha} v_{j}\right) & \leq\left\|D_{\theta}^{\alpha} v_{k}\right\|\left\|D_{\theta+\pi}^{\alpha} v_{j}\right\| \\
& \leq C\left\|v_{k}\right\|_{A}\left\|v_{j}\right\|_{A} \leq C\left\|v_{k}\right\|_{A}^{2}+C\left\|v_{j}\right\|_{A}^{2}, \text { if } \operatorname{dist}\left(\Omega_{k}^{\prime}, \Omega_{j}^{\prime}\right)<H
\end{aligned}
$$

(by Lemma 2.7 and (3.2));

$$
\left(D_{\theta}^{\alpha} v_{k}, D_{\theta+\pi}^{\alpha} v_{j}\right) \leq \frac{C}{l^{0.5+\alpha}}\left\|v_{k}\right\|\left\|_{A}\right\| v_{j} \|_{A} \leq \frac{C}{l^{0.5+\alpha}}\left(\left\|v_{k}\right\|_{A}^{2}+\left\|v_{j}\right\|_{A}^{2}\right), \text { if } \operatorname{dist}\left(\Omega_{k}^{\prime}, \Omega_{j}^{\prime}\right) \geq l H
$$

(by Lemma 4.2).

Fixing $i \in S$, we write

$$
S_{i}=\left\{j \in S: \operatorname{dist}\left(\Omega_{i}^{\prime}, \Omega_{j}^{\prime}\right)<H\right\}, \quad S_{i}^{\prime}=\left\{j \in S: \operatorname{dist}\left(\Omega_{i}^{\prime}, \Omega_{j}^{\prime}\right) \geq H\right\} .
$$

It is easy to see that

$$
\begin{aligned}
& \text { the sum of the terms containing } v_{i} \text { on the left hand of (4.16) } \\
& \leq \sum_{j \in S}\left|\left(D_{\theta}^{\alpha} v_{i}, D_{\theta+\pi}^{\alpha} v_{j}\right)\right|+\sum_{j \in S}\left|\left(D_{\theta}^{\alpha} v_{j}, D_{\theta+\pi}^{\alpha} v_{i}\right)\right| \\
& =\sum_{j \in S_{i}}\left|\left(D_{\theta}^{\alpha} v_{i}, D_{\theta+\pi}^{\alpha} v_{j}\right)\right|+\sum_{j \in S_{i}}\left|\left(D_{\theta}^{\alpha} v_{j}, D_{\theta+\pi}^{\alpha} v_{i}\right)\right| \\
& \quad+\sum_{j \in S_{i}^{\prime}}\left|\left(D_{\theta}^{\alpha} v_{i}, D_{\theta+\pi}^{\alpha} v_{j}\right)\right|+\sum_{j \in S_{i}^{\prime}}\left|\left(D_{\theta}^{\alpha} v_{j}, D_{\theta+\pi}^{\alpha} v_{i}\right)\right| .
\end{aligned}
$$

We know that

$$
\operatorname{card}\left(S_{i}\right) \leq C,
$$

where $\operatorname{card}(S)$ denotes the number of elements contained in $S, C$ is a positive constant dependent of $J^{C}$. By (4.21) and (4.17), we have

$$
\sum_{j \in S_{i}}\left|\left(D_{\theta}^{\alpha} v_{i}, D_{\theta+\pi}^{\alpha} v_{j}\right)\right|+\sum_{j \in S_{i}}\left|\left(D_{\theta}^{\alpha} v_{j}, D_{\theta+\pi}^{\alpha} v_{i}\right)\right| \leq C\left\|v_{i}\right\|_{A}^{2}+\text { terms which do not contain } v_{i} .
$$



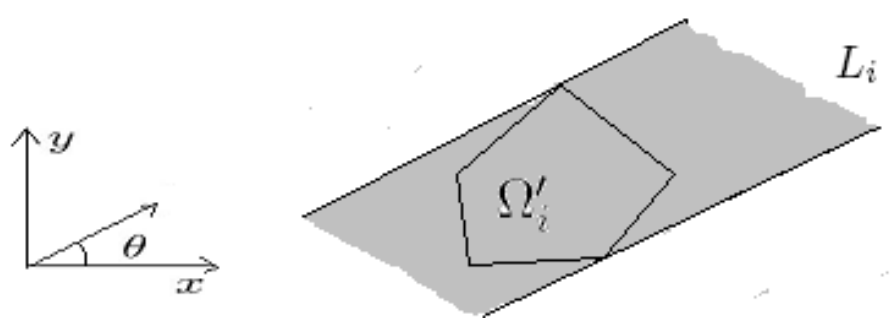

Figure 2:

As figure 2 shows, $L_{i}$, containing $\Omega_{i}^{\prime}$, denotes the shaded bar area whose edges are parallel to the vector $(\cos (\theta), \sin (\theta))$ and go through $\bar{\Omega}_{i}^{\prime}$. It is not hard to see that $\left(D_{\theta}^{\alpha} v_{i}, D_{\theta+\pi}^{\alpha} v_{j}\right)=0$ if $\Omega_{j}^{\prime} \cap L_{i}=\emptyset$, and for positive integer $l$, the number of the elements of the set $S_{i, l}^{\prime}=\left\{\Omega_{k}^{\prime} ; \Omega_{k}^{\prime} \cap L_{i} \neq\right.$ $\left.\emptyset, l H \leq \operatorname{dist}\left(\Omega_{k}^{\prime}, \Omega_{i}^{\prime}\right)<(l+1) H\right\}$ is bounded by a positive $C$ which is a positive constant dependent of $J^{C}$. And then using (4.18), we have

$$
\begin{aligned}
& \sum_{j \in S_{i}^{\prime}}\left|\left(D_{\theta}^{\alpha} v_{i}, D_{\theta+\pi}^{\alpha} v_{j}\right)\right|+\sum_{j \in S_{i}^{\prime}}\left|\left(D_{\theta}^{\alpha} v_{j}, D_{\theta+\pi}^{\alpha} v_{i}\right)\right| \\
\leq & \sum_{l \geq 1} \sum_{j \in S_{i, l}^{\prime}}\left|\left(D_{\theta}^{\alpha} v_{i}, D_{\theta+\pi}^{\alpha} v_{j}\right)\right|+\sum_{l \geq 1} \sum_{j \in S_{i, l}^{\prime}}\left|\left(D_{\theta}^{\alpha} v_{j}, D_{\theta+\pi}^{\alpha} v_{i}\right)\right| \\
\leq & \sum_{l=1}^{\infty} \frac{C}{l^{0.5+\alpha}}\left\|v_{i}\right\|_{A}^{2}+\text { terms which do not contain } v_{i} \\
\leq & C\left\|v_{i}\right\|_{A}^{2}+\text { terms which do not contain } v_{i} .
\end{aligned}
$$

From above analysis, using (4.17) and (4.18) to enlarge the correspondent terms of the left hand of (4.16), we may show that the inequality (4.16) holds with constant $C$ being independent of $\theta$.

Lemma 4.4. There exist linear operators $\kappa_{i}(i=0,1, \ldots, J), \kappa_{0}$ maps $\tilde{H}_{0}^{1}(\Omega)$ into $V_{0}, \kappa_{i}(i=$ $1, \ldots, J)$ maps $\tilde{H}_{0}^{1}(\Omega)$ and $V_{h}$ into $\tilde{H}_{0}^{1}\left(\Omega_{i}^{\prime}\right)$ and $V_{i}$ respectively, such that for any $v \in \tilde{H}_{0}^{1}(\Omega)$,

$$
\begin{gathered}
v=\sum_{i=0}^{J} \kappa_{i} v \\
\sum_{i=0}^{J}\left\|\kappa_{i} v\right\|^{2} \leq C\|v\|^{2}
\end{gathered}
$$

and

$$
\sum_{i=0}^{J}\left\|\kappa_{i} v\right\|_{\tilde{H}^{1}(\Omega)}^{2} \leq C\left(1+\frac{H}{\delta}\right)\|v\|_{\tilde{H}^{1}(\Omega)}^{2},
$$

where the constant $C$ depends on $J^{C}$. 
Proof. We employ the symbols used in the Lemma 3.12 of [28]. First let $\tilde{I}^{H}: H_{0}^{1}(\Omega) \rightarrow V_{H}$ be a quasi-interpolation operator, which is defined as, for the node $y$ in the coarse triangulation $\Gamma_{H}$,

$$
\left(\tilde{I}^{H} w\right)(y)= \begin{cases}0, & y \in \partial \Omega \\ \left|\omega_{y}\right|^{-1} \int_{\omega_{y}} w(x) d x, & \text { otherwise }\end{cases}
$$

where $\omega_{y}$ denotes the union of elements in $\Gamma_{H}$ that share $y,\left|\omega_{y}\right|$ denotes the measure of $\omega_{y}$. Let $I^{h}$ be the piecewise linear interpolation on the fine triangulation $\Gamma_{h}, Q^{h}$ be the $L^{2}$ projector from $\tilde{H}_{0}^{1}(\Omega)$ into $V_{h}$, and $\theta_{i}(i=1, \ldots, J)$ be the modified unit partitions of $\left\{\Omega_{i}^{\prime}\right\}_{i=1}^{J}$ (see (3.7) in [28]) which are piecewise linear functions on $\Gamma_{h}$, satisfying $\sum_{i=1}^{J} \theta_{i}=1, \max _{1 \leq i \leq J}\left\|\nabla \theta_{i}(x)\right\|_{\infty} \leq \frac{C}{\delta}$ and $\operatorname{supp}\left(\theta_{i}\right)=\Omega_{i}^{\prime}$.

Take $\kappa_{0}=\tilde{I}^{H}$ and define $\kappa_{i}, i=1,2, \ldots, J$ as follows: for $w \in \tilde{H}_{0}^{1}(\Omega)$,

$$
\kappa_{i} w:=I^{h}\left(\theta_{i} Q^{h}\left(I-\kappa_{0}\right) w\right)+\theta_{i}\left(I-Q^{h}\right) w .
$$

It is not hard to see that $\kappa_{0}$ maps $\tilde{H}_{0}^{1}(\Omega)$ into $V_{0}, \kappa_{i}(i=1, \ldots, J)$ maps $\tilde{H}_{0}^{1}(\Omega)$ and $V_{h}$ into $\tilde{H}_{0}^{1}\left(\Omega_{i}^{\prime}\right)$ and $V_{i}$ respectively, and (4.24) holds. Furthermore, define $\tilde{v}_{i}:=I^{h}\left(\theta_{i} Q^{h}\left(I-\kappa_{0}\right) v\right), \tilde{\tilde{v}}_{i}:=$ $\theta_{i}\left(I-Q^{h}\right) v, i=1,2, \ldots, J$. It is easy to see that

$$
\begin{gathered}
\sum_{i=0}^{J}\left\|\kappa_{i} v\right\|^{2} \leq\left\|\kappa_{0} v\right\|^{2}+C \sum_{i=1}^{J}\left\|\tilde{v}_{i}\right\|^{2}+C \sum_{i=1}^{J}\left\|\tilde{\tilde{v}}_{i}\right\|^{2}, \\
\sum_{i=0}^{J}\left\|\kappa_{i} v\right\|_{\tilde{H}^{1}(\Omega)}^{2} \leq\left\|\kappa_{0} v\right\|_{\tilde{H}^{1}(\Omega)}^{2}+C \sum_{i=1}^{J}\left\|\tilde{v}_{i}\right\|_{\tilde{H}^{1}(\Omega)}^{2}+C \sum_{i=1}^{J}\left\|\tilde{\tilde{v}}_{i}\right\|_{\tilde{H}^{1}(\Omega)}^{2} .
\end{gathered}
$$

In order to show that (4.25) and (4.26) hold, it suffices to show that

$$
\begin{gathered}
\left\|\kappa_{0} v\right\|^{2} \leq C\|v\|^{2} \\
\sum_{i=1}^{J}\left\|\tilde{v}_{i}\right\|^{2} \leq C\|v\|^{2}, \sum_{i=1}^{J}\left\|\tilde{\tilde{v}}_{i}\right\|^{2} \leq C\|v\|^{2}, \\
\left\|\kappa_{0} v\right\|_{\tilde{H}^{1}(\Omega)}^{2} \leq C\|v\|_{\tilde{H}^{1}(\Omega)}^{2},
\end{gathered}
$$

and

$$
\sum_{i=1}^{J}\left\|\tilde{v}_{i}\right\|_{\tilde{H}^{1}(\Omega)}^{2} \leq C\left(1+\frac{H}{\delta}\right)\|v\|_{\tilde{H}^{1}(\Omega)}^{2}, \quad \sum_{i=1}^{J}\left\|\tilde{\tilde{v}}_{i}\right\|_{\tilde{H}^{1}(\Omega)}^{2} \leq C\left(1+\frac{H}{\delta}\right)\|v\|_{\tilde{H}^{1}(\Omega)}^{2} .
$$

It is known that [28]

$$
\left\|w-Q^{h} w\right\|+h\left\|Q^{h} w\right\|_{\tilde{H}^{1}(\Omega)} \leq C h\|w\|_{\tilde{H}^{1}(\Omega)}, \quad w \in \tilde{H}_{0}^{1}(\Omega) .
$$

By Lemma 3.6 in [28], we know

$$
\left\|w-\tilde{I}^{H} w\right\|+H\left\|\tilde{I}^{H} w\right\|_{\tilde{H}^{1}(\Omega)} \leq C H\|w\|_{\tilde{H}^{1}(\Omega)}, \quad w \in \tilde{H}_{0}^{1}(\Omega) .
$$

By a similar proof of Lemma 3.9 in [28], we may get

$$
\left\|I^{h} w\right\|_{\tilde{H}^{1}(\Omega)} \leq C\|w\|\left\|_{\tilde{H}^{1}(\Omega)}, \quad\right\| I^{h} w\|\leq C\| w \|,
$$


here $w$ is a continuous piecewise quadratic function on $\Gamma_{h}$.

Then (4.29) and (4.31) are a direct consequence of (4.35), meanwhile (4.30) and (4.32) can be proved by using (4.33), (4.34) and (4.35) (Please refer to Lemma 3.12 in 28] for details).

Next, we shall present the main results for the preconditioner system (3.5).

Lemma 4.5. The operator $P_{h}$ is symmetric positive definite with respect to the inner product $(\cdot, \cdot)_{A}$.

Proof. For the proof, we refer to section 2.3 in [28].

Lemma 4.6. For $v \in V_{h}$, we have

$$
\left(P_{h} v, v\right)_{A} \leq C(v, v)_{A} .
$$

Proof. By the definition of $P_{0}$, we have

$$
\left(P_{0} v, v\right)_{A}=\left(P_{0} v, P_{0} v\right)_{A} \leq(v, v)_{A} .
$$

We recall the index set $I_{i}\left(i=1,2, \ldots, J^{C}\right)$ : for any $j, k \in I_{i}$ with $j \neq k, \Omega_{j}^{\prime} \cap \Omega_{k}^{\prime}=\emptyset$, so it is easy to see that

$$
\left(P_{j} v, w\right)_{A}=\left(\tilde{P}_{i} v, w\right)_{A}, \quad \forall w \in V_{j}, j \in I_{i}, v \in V_{h} .
$$

For any $w \in \tilde{V}_{i}$, decompose it as $w=\sum_{j \in I_{i}} w_{j}, w_{j} \in V_{j}$. Then by (4.37), we have

$$
\sum_{j \in I_{i}}\left(P_{j} v, w_{j}\right)_{A}=\sum_{j \in I_{i}}\left(\tilde{P}_{i} v, w_{j}\right)_{A}=\left(\tilde{P}_{i} v, w\right)_{A}, \quad v \in V_{h} .
$$

Taking $w_{j}=P_{j} v$ in the above equation, and using Cauchy-Schwarz inequality and Lemma 4.3, we may obtain

$$
\begin{aligned}
\sum_{j \in I_{i}}\left(P_{j} v, P_{j} v\right)_{A} & =\left(\tilde{P}_{i} v, \sum_{j \in I_{i}} P_{j} v\right)_{A} \\
& \leq\left\|\tilde{P}_{i} v\right\|_{A}\left\|\sum_{j \in I_{i}} P_{j} v\right\|_{A} \lesssim\left\|\tilde{P}_{i} v\right\|_{A}\left(\sum_{j \in I_{i}}\left\|P_{j} v\right\|_{A}^{2}\right)^{1 / 2},
\end{aligned}
$$

which is

$$
\sum_{j \in I_{i}}\left(P_{j} v, P_{j} v\right)_{A} \lesssim\left\|\tilde{P}_{i} v\right\|_{A}^{2}
$$

It is easy to see that

$$
\left(\tilde{P}_{i} v, v\right)_{A}=\left(\tilde{P}_{i} v, \tilde{P}_{i} v\right)_{A} \leq(v, v)_{A}
$$

and

$$
\sum_{j \in I_{i}}\left(P_{j} v, P_{j} v\right)_{A} \lesssim\left\|\tilde{P}_{i} v\right\|_{A}^{2} \leq(v, v)_{A} .
$$

Combining (4.42) with (4.36), we arrive at

$$
\begin{aligned}
\left(P_{h} v, v\right)_{A} & =\left(P_{0} v, P_{0} v\right)_{A}+\left(P_{1} v, P_{1} v\right)_{A}+\cdots+\left(P_{J} v, P_{J} v\right)_{A} \\
& \leq\left(P_{0} v, P_{0} v\right)_{A}+\sum_{j \in I_{1}}\left(P_{j} v, P_{j} v\right)_{A}+\cdots+\sum_{j \in I_{J} C}\left(P_{j} v, P_{j} v\right)_{A} \\
& \lesssim\left(1+J^{C}\right)(v, v)_{A} .
\end{aligned}
$$

Then the lemma is proved. 
Lemma 4.7. For $v \in V_{h}$, there exists a decomposition $v=\sum_{i=0}^{J} v_{i}, v_{i} \in V_{i}$, such that

$$
\sum_{i=0}^{J}\left(v_{i}, v_{i}\right)_{A} \leq C\left(1+\frac{H}{\delta}\right)\|v\|_{A}^{2} .
$$

Proof. Let $\kappa_{i}$ be the operators as defined in Lemma 4.4, we now prove the decomposition $v=$ $\sum_{i=0}^{J} v_{i}$ with $v_{i}=\kappa_{i} v$ may satisfy (4.44). Since $\|w\|_{A}^{2} \approx\|w\|_{H^{\alpha}(\Omega)}^{2}$ for $w \in V_{h}$, it suffices to show that

$$
\sum_{i=0}^{J}\left\|\kappa_{i} v\right\|_{H^{\alpha}(\Omega)}^{2} \leq C\left(1+\frac{H}{\delta}\right)\|v\|_{H^{\alpha}(\Omega)}^{2} .
$$

Actually, using the definition of the norms $\|\cdot\|_{\tilde{H}^{\alpha}\left(\mathbb{R}^{2}\right)},\|\cdot\|_{\hat{H}^{\alpha}(\Omega)}$, Lemma 4.1 and Lemma 4.4, we have

$$
\begin{aligned}
& \sum_{i=0}^{J}\left\|\kappa_{i} v\right\|_{H^{\alpha}(\Omega)}^{2} \approx \sum_{i=0}^{J}\left\|\kappa_{i} v\right\|_{\tilde{H}^{\alpha}\left(\mathbb{R}^{2}\right)}^{2} \\
& =\sum_{i=0}^{J} \int_{0}^{\infty} \inf _{w_{i} \in \tilde{H}^{1}\left(\mathbb{R}^{2}\right)}\left(\left\|\kappa_{i} v-w_{i}\right\|_{L^{2}\left(\mathbb{R}^{2}\right)}^{2}+t^{2}\left\|w_{i}\right\|_{\tilde{H}^{1}\left(\mathbb{R}^{2}\right)}^{2}\right) t^{-2 \alpha-1} d t \\
& \leq \sum_{i=0}^{J} \int_{0}^{\infty} \inf _{w_{i} \in \tilde{H}_{0}^{1}(\Omega)}\left(\left\|\kappa_{i} v-w_{i}\right\|_{L^{2}\left(\mathbb{R}^{2}\right)}^{2}+t^{2}\left\|w_{i}\right\|_{\tilde{H}^{1}\left(\mathbb{R}^{2}\right)}^{2}\right) t^{-2 \alpha-1} d t \\
& =\sum_{i=0}^{J} \int_{0}^{\infty} \inf _{w_{i} \in \tilde{H}_{0}^{1}(\Omega)}\left(\left\|\kappa_{i} v-w_{i}\right\|_{L^{2}(\Omega)}^{2}+t^{2}\left\|w_{i}\right\|_{\tilde{H}^{1}(\Omega)}^{2}\right) t^{-2 \alpha-1} d t \\
& =\int_{0}^{\infty} \inf _{w_{0}, \ldots, w_{J} \in \tilde{H}_{0}^{1}(\Omega)} \sum_{i=0}^{J}\left(\left\|\kappa_{i} v-w_{i}\right\|_{L^{2}(\Omega)}^{2}+t^{2}\left\|w_{i}\right\|_{\tilde{H}^{1}(\Omega)}^{2}\right) t^{-2 \alpha-1} d t \\
& \leq \int_{0}^{\infty} \inf _{w \in \tilde{H}_{0}^{1}(\Omega)} \sum_{i=0}^{J}\left(\left\|\kappa_{i} v-\kappa_{i} w\right\|_{L^{2}(\Omega)}^{2}+t^{2}\left\|\kappa_{i} w\right\|_{\tilde{H}^{1}(\Omega)}^{2}\right) t^{-2 \alpha-1} d t \\
& \lesssim\left(1+\frac{H}{\delta}\right) \int_{0}^{\infty} \inf _{w \in \tilde{H}_{0}^{1}(\Omega)}\left(\|v-w\|_{L^{2}(\Omega)}^{2}+t^{2}\|w\|_{\tilde{H}^{1}(\Omega)}^{2}\right) t^{-2 \alpha-1} d t \\
& =\left(1+\frac{H}{\delta}\right)\|v\|_{\hat{H}^{\alpha}(\Omega)}^{2} \approx\left(1+\frac{H}{\delta}\right)\|v\|_{\tilde{H}^{\alpha}(\Omega)}^{2} .
\end{aligned}
$$

Moreover,

$$
\begin{aligned}
\|v\|_{\tilde{H}^{\alpha}(\Omega)}^{2} & =\int_{0}^{\infty} \inf _{w \in \tilde{H}^{1}(\Omega)}\left(\|v-w\|_{L^{2}(\Omega)}^{2}+t^{2}\|w\|_{\tilde{H}^{1}(\Omega)}^{2}\right) t^{-2 \alpha-1} d t \\
& \leq \int_{0}^{\infty} \inf _{w \in \tilde{H}^{1}\left(\mathbb{R}^{2}\right)}\left(\left\|v-\left.w\right|_{\Omega}\right\|_{L^{2}(\Omega)}^{2}+t^{2}\left\|\left.w\right|_{\Omega}\right\|_{\tilde{H}^{1}(\Omega)}^{2}\right) t^{-2 \alpha-1} d t \\
& \leq \int_{0}^{\infty} \inf _{w \in \tilde{H}^{1}\left(\mathbb{R}^{2}\right)}\left(\|v-w\|_{L^{2}\left(\mathbb{R}^{2}\right)}^{2}+t^{2}\|w\|_{\tilde{H}^{1}\left(\mathbb{R}^{2}\right)}^{2}\right) t^{-2 \alpha-1} d t \\
& =\|v\|_{\tilde{H}^{\alpha}\left(\mathbb{R}^{2}\right)} \approx\|v\|_{H^{\alpha}(\Omega)} .
\end{aligned}
$$

Combining (4.47) with (4.46), we may get (4.45). 
Lemma 4.8. For $v \in V_{h}$, we have

$$
(v, v)_{A} \leq C\left(1+\frac{H}{\delta}\right)\left(P_{h} v, v\right)_{A}
$$

Proof. By Lemma 4.7, we know that there exists a decomposition $v=\sum_{i=0}^{J} v_{i}, v_{i} \in V_{i}$ such that (4.44) holds. Then by the Cauchy-Schwarz inequality and (4.44), we have

$$
\begin{aligned}
(v, v)_{A} & =\sum_{i=0}^{J}\left(v, v_{i}\right)_{A}=\sum_{i=0}^{J}\left(P_{i} v, v_{i}\right)_{A} \\
& \leq\left(\sum_{i=0}^{J}\left(P_{i} v, P_{i} v\right)_{A}\right)^{1 / 2}\left(\sum_{i=0}^{J}\left(v_{i}, v_{i}\right)_{A}\right)^{1 / 2} \\
& =\left(\sum_{i=0}^{J}\left(P_{i} v, v\right)_{A}\right)^{1 / 2}\left(\sum_{i=0}^{J}\left(v_{i}, v_{i}\right)_{A}\right)^{1 / 2} \\
& \leq\left(P_{h} v, v\right)_{A}^{1 / 2}\left(C\left(1+\frac{H}{\delta}\right)(v, v)_{A}\right)^{1 / 2}
\end{aligned}
$$

which is Lemma $4.8 \quad \square$.

Theorem 4.9. The condition number of $P_{h}$ satisfies

$$
\operatorname{cond}\left(P_{h}\right) \leq C\left(1+\frac{H}{\delta}\right)
$$

Proof. The theorem follows from Lemma 4.6 and Lemma 4.8.

\section{Numerical results}

We test PCG method with $B_{h}$ as a preconditioner. The tests are carried out by using Matlab software. The stopping criterion is

$$
\left\|u^{k}-u^{k-1}\right\|_{\infty} \leq 10^{-6}
$$

where $u^{k}$ is obtained from $u^{k-1}$ by one step PCG iteration, and $u^{0}=0$. Here we only test the case that $\Omega$ is a square domain. We take the triangulations $\Gamma_{H}, \Gamma_{h}$ as uniform ones as in figure 3 shows. With viewing the one in figure 3 as the coarse triangulation $\Gamma_{H}$, the subdomains $\Omega_{i}$ and $\Omega_{i}^{\prime}$ are taken as the darker and lighter shaded squares respectively.

We give two examples in this paper: one is with the probability measure $\tilde{M}$ having a discrete form and the other is with $\tilde{M}$ being a continuous function. Table 1 and Table 2 list the iterative steps of our PCG methods for Example 5.1 and Example 5.2 respectively. It can be seen that when we fix $\frac{H}{h}=$ constant, for instance $\frac{H}{h}=8$, the convergence rate of our PCG method is optimal, i.e., the iterative steps of the PCG method almost keep to be a constant, which coincides with our theoretical results in this paper. From the tables 1-2, we also find that small overlapping case is a good choice for our domain decomposition methods for the SFPDEs, which is same as the overlapping domain decomposition methods for the integer order PDEs. 


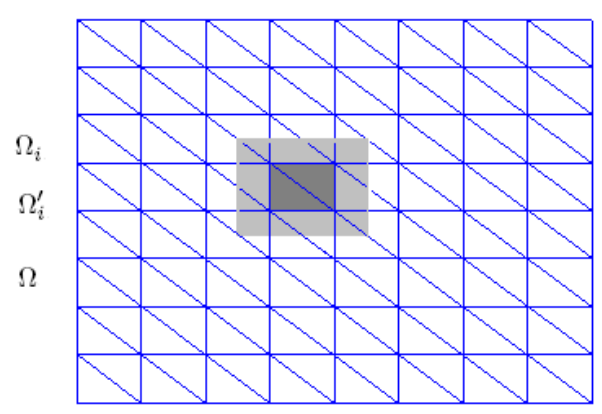

Figure 3: The trangulation and the subdomains

\begin{tabular}{||c|c|c|c||}
\hline$(\mathrm{h}, \mathrm{H})$ & $\delta=h$ & $\delta=2 h$ & $\delta=4 h$ \\
\hline$\left(\frac{2}{64}, \frac{2}{8}\right)$ & 13 & 13 & 14 \\
\hline$\left(\frac{2}{128}, \frac{2}{16}\right)$ & 12 & 11 & 12 \\
\hline$\left(\frac{2}{256}, \frac{2}{32}\right)$ & 10 & 10 & 11 \\
\hline
\end{tabular}

Table 1: The iterative steps of PCG method for Example 5.1

Example 5.1. Let $\Omega=[0,2] \times[0,2]$, the equation is

$$
-\frac{1}{4}\left({ }_{-\infty} D_{x}^{1.5}+{ }_{x} D_{\infty}^{1.5}+{ }_{-\infty} D_{y}^{1.5}+{ }_{y} D_{\infty}^{1.5}\right) u=f(x, y),
$$

where $f(x, y)$ is taken such that the solution is $u=\left(\left(2 x-x^{2}\right)\left(2 y-y^{2}\right)\right)^{4}$,

Example 5.2. Let $\Omega=[0,2] \times[0,2]$, the equation is

$$
-D_{\tilde{M}}^{1.5} u=f(x, y)
$$

where $\tilde{M}(\theta)=1, \theta \in[0,2 \pi)$, and $f(x, y)$ is taken as in Example 5.1 .

\section{Appendix}

In this appendix, we shall present the definitions of the fractional integrals and derivatives in one dimension case, and their corresponding properties, which are used in this paper. The related results can also be found in [21,24]. For function $v(x), x \in(a, b)$, where $a$ may be taken as $-\infty$, and $b$ as $+\infty$, the left and right Riemann-Liouville fractional Integrals of order $\mu>0$ are defined respectively by

$$
{ }_{a} D_{x}^{-\mu} v(x)=\frac{1}{\Gamma(\mu)} \int_{a}^{x}(x-s)^{\mu-1} v(s) d s, \quad{ }_{x} D_{b}^{-\mu} v(x)=\frac{1}{\Gamma(\mu)} \int_{x}^{b}(s-x)^{\mu-1} v(s) d s .
$$

\begin{tabular}{||c|c|c|c||}
\hline$(\mathrm{h}, \mathrm{H})$ & $\delta=h$ & $\delta=2 h$ & $\delta=4 h$ \\
\hline$\left(\frac{2}{64}, \frac{2}{8}\right)$ & 13 & 13 & 13 \\
\hline$\left(\frac{2}{128}, \frac{2}{16}\right)$ & 13 & 13 & 13 \\
\hline$\left(\frac{2}{256}, \frac{2}{32}\right)$ & 13 & 12 & 13 \\
\hline
\end{tabular}

Table 2: The iterative steps of PCG method for Example 5.2 
The left and right Riemann-Liouville fractional derivatives of order $\mu>0$ are defined respectively by

$$
\begin{gathered}
{ }_{a} D_{x}^{\mu} v(x)=D_{a}^{n} D_{x}^{-(n-\mu)} v(x)=\frac{1}{\Gamma(n-\mu)} \frac{d^{n}}{d x^{n}} \int_{a}^{x}(x-s)^{n-\mu-1} v(s) d s, \\
{ }_{x} D_{b}^{\mu} v(x)=(-D)^{n}{ }_{x} D_{b}^{-(n-\mu)} v(x)=\frac{(-1)^{n}}{\Gamma(n-\mu)} \int_{x}^{b}(s-x)^{n-\mu-1} v(s) d s,
\end{gathered}
$$

where $n$ is an integer such that $n-1 \leq \mu<n$. For $p>0, k$ is an integer satisfying $k-1 \leq p<k$. If ${ }_{a} D_{x}^{p} v(x)$ is integrable, then

$$
{ }_{a} D_{x}^{-p}{ }_{a} D_{x}^{p} v(x)=v(x)-\sum_{j=1}^{k}\left[{ }_{a} D_{x}^{p-j} v(x)\right] \mid{ }_{x=a} \frac{(x-a)^{p-j}}{\Gamma(p-j+1)} .
$$

(A.4) is from (2.108) in [21].

\section{References}

[1] R. A. Adams, Sobolev Spaces, Academic Press, New York, 1975.

[2] B. Beumer, M. Kovcs and M. M. Meerschaert, Numerical solutions for fractional reaction diffusion equations, Comput. Math. Appl., 55 (2008), pp. 2212-2226.

[3] R. Chan and X. Jin, An Introduction to Iterative Toeplitz Solvers, SIAM, Philadelphia, 2007.

[4] R. Chan and M. Ng, Conjugate gradient methods for Toeplitz systems, SIAM Rev., 38 (1996), pp. 427-482.

[5] P. G. Ciarlet, The Finite Element Methods for Elliptic Problems, North-Holland, New York, 1978.

[6] W. Deng, Finite element method for the space and time fractional Fokker-Planck equation, SIAM J. Numer. Anal. 47 (2008), pp. 204-226.

[7] V. J. Ervin, N. Heuer and J. P. Roop, Numerical approximation of a time dependent, nonlinear, space-fractional diffusion equation, SIAM J. Numer. Anal. 45 (2007), pp. 572-591.

[8] V. J. Ervin and J. P. Roop, Variational solution of the fractional advection dispersion equation on bounded domains in $R^{d}$, Numer. Meth. P.D.E., 23 (2007), pp. 256-281.

[9] Y. Jiang and X. Xu, Multigrid methods for space fractional partial differential equations, available online at http://arxiv.org/pdf/1501.01475.pdf.

[10] S. L. Lei and H. W Sun, A circulant preconditioner for fractional diffusion equations, J. Comput. Phys., 242 (2013), pp. 715-725.

[11] X. Li and C. Xu, The existence and uniqueness of the week solution of the space-time fractional diffusion equation and a spectral method approximation, Commun. Comput. Phys., 8 (2010), pp. 1016-1051.

[12] F. R. Lin, S. W. Yang and X. Q. Jin, Preconditioned iterative methods for fractional diffusion equation, J. Comput. Phys., 256 (2014), pp. 109-117.

[13] J. L. Lions, E. Magenes, Nonhomogeneous Boundary Value Problems and Applications I, Springer, Berlin (1972) 
[14] F. Liu, V. Anh and I. Turner, Numerical solution of the space fractional Fokker-Planck equation, J. Comput. Appl. Math., 166 (2004), pp. 209-219.

[15] M. M. Meerschaert, D. A. Benson and B. Baumer, Multidimensional advection and fractional dispersion, Phys. Rev. E., 59 (1999), pp. 5026-5028.

[16] M. M. Meerschaert, J. Mortensen and H. P. Scheffler, Vector Grunwald formula for fractional derivatives, Fract. Calc. Appl. Anal., 7 (2004), pp. 61-82.

[17] M. M. Meerschaert and C. Tadjeran, Finite difference approximations for two-sided space-fractional partial differential equations, Appl. Numer. Math., 56 (2006), pp. 80-90.

[18] M. M. Meerschaert, H. P. Scheffler and C. Tadjeran, Finite difference methods for two-dimensional fractional dispersion equation, J. Comput. Phys., 211 (2006), pp. 249-261.

[19] R. Metzler and J. Klafter, The random walk's guide to anomalous diffusion: A fractional dynamics approach, Phys. Rep., 339 (2000), pp. 1-77.

[20] H. Pang and H. Sun, Multigrid method for fractional diffusion equations, J. Comput. Phys., 231 (2012), pp. 693-703.

[21] I. Podlubny, Fractional Differential Equations, Academic Press, New York, 1999.

[22] J. Roop, Computational aspects of FEM approximation of fractional advection dispersion equations on bounded domains in R2, J. Comput. Appl. Math., 193 (2006), pp. 243-268.

[23] W. Rudin, Real and Complex Analysis, McGraw-Hill, New York, 1987.

[24] S. G. Samko, A. A. Kilbas and O. I. Marichev, Fractional Integrals and Derivatives: Theory and Applications, Gordon and Breach, New York, 1993.

[25] E. Sousa, Finite difference approximates for a fractional advection diffusion problem, J. Comput. Phys. 228 (2009), pp. 4038-4054.

[26] C. Tadjeran, M. M. Meerschaert and H. P. Scheffler, A second-order accurate numerical approximation for the fractional diffusion equation, J. Comput. Phys., 213 (2006), pp. 205-213.

[27] L. Tartar, An Introduction to Sobolev Spaces and Interpolation Spaces, Lecture Notes of the Unione Matematica Italiana 3, Springer-Verlag, Berlin Heidelberg, 2007.

[28] A. Toselli, O. Widlund, Domain Decomposition Methods-Algorithms and Theory, Springer-Verlag, Berlin Heidelberg, 2005.

[29] H. Wang, K. Wang and T. Sircar, Adirect $O\left(N \log ^{2} N\right)$ finite difference method for fractional diffusion equations, J. Comput. Phys., 229 (2010), pp. 8095-8104.

[30] H. Wang and K. Wang, An $O\left(N \log ^{2} N\right)$ alternating-direction finite difference method for twodimensional fractional diffusion equations, J. Comput. Phys., 21 (2011), pp 7830-7839.

[31] H. Wang and N. Du, Fast alternating-direction finite difference methods for three-dimensional spacefractional diffusion equations, J. Comput. Phys., 258 (2014), pp 305-318.

[32] K. Wang and $\mathrm{H}$. Wang, A fast characteristic finite difference method for fractional advection-diffusion equations, Adv. Water Resour, 34 (2011), pp. 810-816.

[33] H. Wang and N. Du, A superfast-preconditioned iterative method for steady-state space-fractional diffusion equations, J. Comput. Phys., 240 (2013), pp. 49-57. 
[34] H. Wang and N. Du, A fast finite difference method for three-dimensional time-dependent spacefractional diffusion equations and its efficient implementation, J. Comput. Phys., 253 (2013), pp. $50-63$.

[35] J. C. Xu, Iterative methods by space decomposition and subspace correction, SIAM Rev., 34 (1992), pp. 581-613.

[36] Z. Zhou and H. Wu, Finite element multigrid method for the boundary value problem of fractional advection dispersion equation, J. Appl. Math., 2013, Article ID 385463, 8 pages. 